\title{
Modeling and Simulation of a Resonant-Cavity-Enhanced InGaAs/GaAs Quantum Dot Photodetector
}

\author{
W. W. Wang, F. M. Guo, and Y. Q. Li \\ Shanghai Key Laboratory of Multidimensional Information Processing, Key Laboratory of Polar Materials \& Devices, \\ School of Information Science Technology, East China Normal University, No. 500, Dong Chuan Road, Shanghai 200241, China \\ Correspondence should be addressed to F. M. Guo; fmguo@ee.ecnu.edu.cn
}

Received 27 November 2014; Accepted 25 December 2014

Academic Editor: Wen Lei

Copyright (C) 2015 W. W. Wang et al. This is an open access article distributed under the Creative Commons Attribution License, which permits unrestricted use, distribution, and reproduction in any medium, provided the original work is properly cited.

We simulated and analyzed a resonant-cavity-enhancedd InGaAs/GaAs quantum dot n-i-n photodiode using Crosslight Apsys package. The resonant cavity has a distributed Bragg reflector (DBR) at one side. Comparing with the conventional photodetectors, the resonant-cavity-enhanced photodiode (RCE-PD) showed higher detection efficiency, faster response speed, and better wavelength selectivity and spatial orientation selectivity. Our simulation results also showed that when an AlAs layer is inserted into the device structure as a blocking layer, ultralow dark current can be achieved, with dark current densities $0.0034 \mathrm{~A} / \mathrm{cm}$ at $0 \mathrm{~V}$ and $0.026 \mathrm{~A} / \mathrm{cm}$ at a reverse bias of $2 \mathrm{~V}$. We discussed the mechanism producing the photocurrent at various reverse bias. A high quantum efficiency of $87.9 \%$ was achieved at resonant wavelength of $1030 \mathrm{~nm}$ with a FWHM of about $3 \mathrm{~nm}$. We also simulated InAs QD RCE-PD to compare with InGaAs QD. At last, the photocapacitance characteristic of the model has been discussed under different frequencies.

\section{Introduction}

Low dimensional III-V semiconductor nanostructures have been widely studied on their electronic and optical properties for device applications. Among the nanostructures, the resonant-cavity-enhanced photodetectors (RCE-PDs) have been extensively studied due to their novel physical mechanism and special device applications over the past two decades [1-7]. A RCE-PD is usually constructed by placing multiple active layers at the peak positions of a standing wave in a resonant cavity, sandwiched between two distributed Bragg reflectors (DBRs).

The DBR mirrors are usually made of quarter wave stacks with a periodic modulation of refractive indices. Metals are usually not used as mirrors because they do not provide wavelength selectivity [2]. RCE-PDs also have the ability of wavelength selectivity. When light is incident on the detector, the light will enter the cavity and interfere in the internal cavity which consisted of two mirrors if the wavelength and the cavity mode match. So optical field is enhanced and more light will be absorbed, generating more carriers in the cavity. Compared with the conventional detectors, the responsivity is greatly improved. This makes the RCE-PD with high detection efficiency, fast response speed, ability to work in the absence of bias voltage, and their wavelength selectivity and spatial orientation selectivity especially suitable for optical fiber communication system that requires high wavelength accuracy.

$1.06 \mu \mathrm{m}$ is a specific wavelength used in Nd:YAG laser systems. These systems have been widely deployed in industrial manufacturing, medicine, remote sensing, space communication, and so forth. To achieve high sensitive detection at this wavelength, resonant-cavity-enhanced- (RCE-) PDs with quantum dots (QDs) as absorption layers were explored to reach a peak quantum efficiency of 65-75\% [8-10]. However, DBR mirrors make it difficult for the device to extract photogenerated carriers. To solve this problem, Bennett et al. and Sun et al. fabricated an InGaAs/GaAs quantum dot $n$-i-n RCE-PDs with only a bottom DBR mirror which still has the resonant coupling nature and achieved peak photoresponsivity of $0.75 \mathrm{~A} / \mathrm{W}$ at $1.4 \mathrm{~V}[11,12]$.

\section{Materials and Methods}

The device structure to be imported into APSYS was constructed according to the structure in [12]. As shown in 


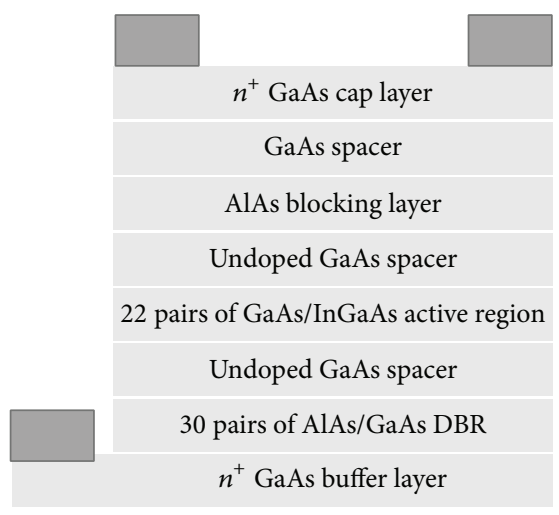

(a)

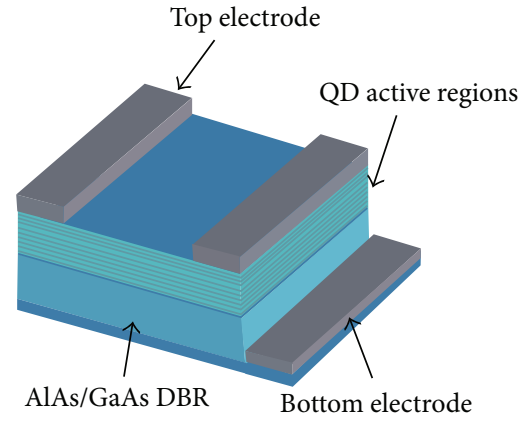

(b)

FIGURE 1: (a) The schematic diagram of the resonant-cavity-enhanced photodetector (RCE-PD). (b) The simplified 3D view of the device constructed.

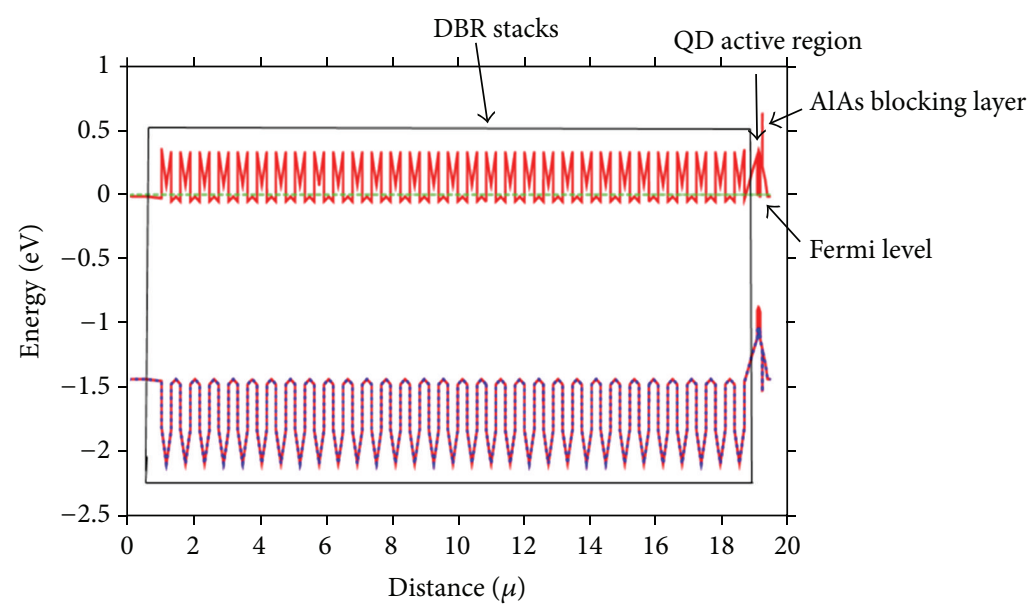

FIGURE 2: Energy band diagram profile along epitaxial growth direction under equilibrium.

Figure 1(a), it consists of a Si-doped $1 \mu \mathrm{m}$ GaAs buffer layer and a DBR (n-doped) with 30 pairs of quarter-wavelength $(\lambda / 4) \mathrm{GaAs} / \mathrm{AlAs}$ layers. The active region is $380 \mathrm{~nm}$ thick undoped GaAs spacer and 23 periods of $6 \mathrm{~nm}-\mathrm{In} 0.5 \mathrm{Ga} 0.5 \mathrm{As}$ QDs layers. Finally, the top layers in sequence are $50 \mathrm{~nm}$ undoped GaAs spacer, $3 \mathrm{~nm}$ thick AlAs blocking layer, $157 \mathrm{~nm}$ GaAs spacer, and a $175 \mathrm{~nm} n^{+}$GaAs cap layer. The doping concentration for all the $n^{+}$layers is $1 \times 10^{18} \mathrm{~cm}^{-3}$. QD sheet density of $3 \times 10^{10} \mathrm{~cm}^{-2}$ and a square $(80 \mu \mathrm{m} \times 80 \mu \mathrm{m})$ opening was left on the top for optical access. The temperature is set to be room temperature. Plotted in Figure 1(b) is a simplified 3D structure view of the device constructed by Lumerical software. The energy band diagram of the device at thermal equilibrium along the epitaxial growth direction is plotted in Figure 2.

Figure 3(a) is the simulated QD PL spectrum at room temperature. The peak at $1.13 \mu \mathrm{m}$ is emission from the quantum dots and $960 \mathrm{~nm}$ is from the wetting layer $[9,13]$. Figure $3(b)$ is the gain of the absorption layer at different incident wavelengths. The largest gain appearing at $1.13 \mu \mathrm{m}$ indicated that electron-hole pairs generated in the active region reached a maximum.

\section{Results and Discussion}

Figure 4(a) is the current-voltage characteristics of the InGaAs QD RCE photodetector simulated at $1030 \mathrm{~nm}$ wavelength. The suppressed dark current in the $n-i-n$ structure is attributed to the blocking effect by the AlAs layer. The current increases as the reverse bias increases. In the process of optical transition, the excited electrons from the conduction band and the excited holes from the valence band will form excitons in QDs. The quantum dots can capture the excitons very effectively. Potential barrier prevents the electron-hole from moving in the opposite direction. Thus the tunneling probability through the potential barrier is very small. Exciton ionization is mainly thermal ionization. The electron can escape from the quantum dots producing a photocurrent at a certain temperature. The escape of electrons and holes changes electric potential, resulting in a decrease of the effective potential barrier, which makes bound electrons and holes in the quantum dots tunnel through the barrier, thus increasing the photocurrent. It is observed that the dark current increases a little at $0.5 \mathrm{~V}$ or less. When the bias is $0.5 \mathrm{~V}$, photocurrent is only $0.02 \mathrm{~A} / \mathrm{cm}$. The slow increase of photocurrent density at lower applied voltage is because 


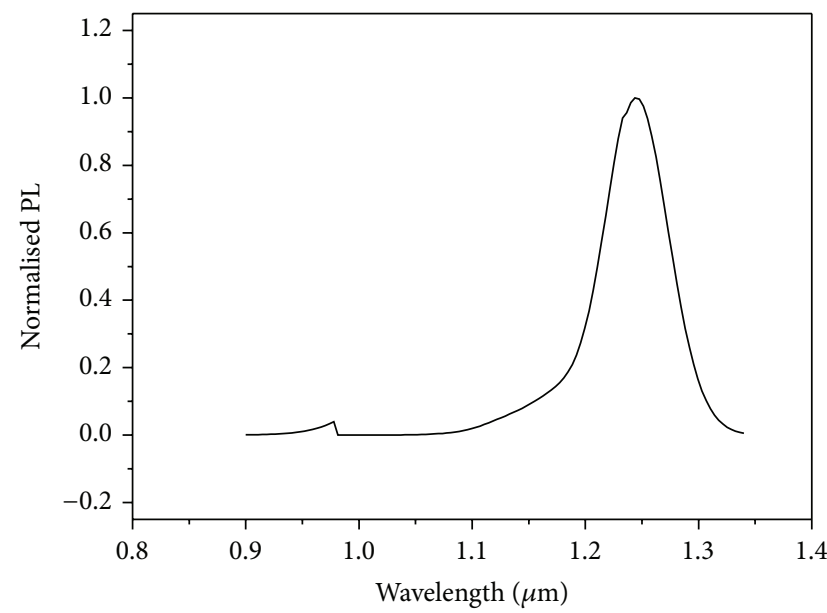

$-T=300 \mathrm{~K}$

(a)

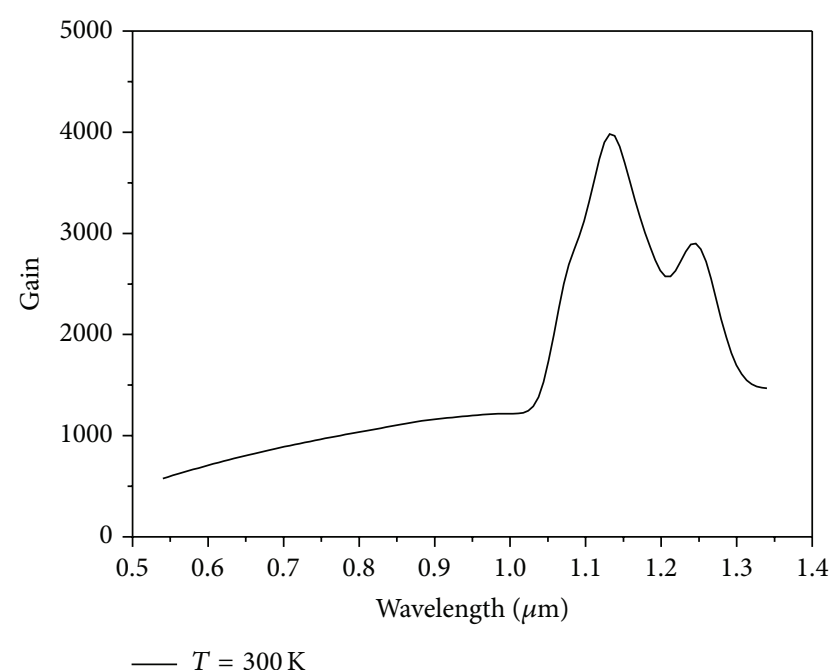

(b)

FIGURE 3: (a) Simulated PL spectrum. (b) Absorption layer gain spectrum.

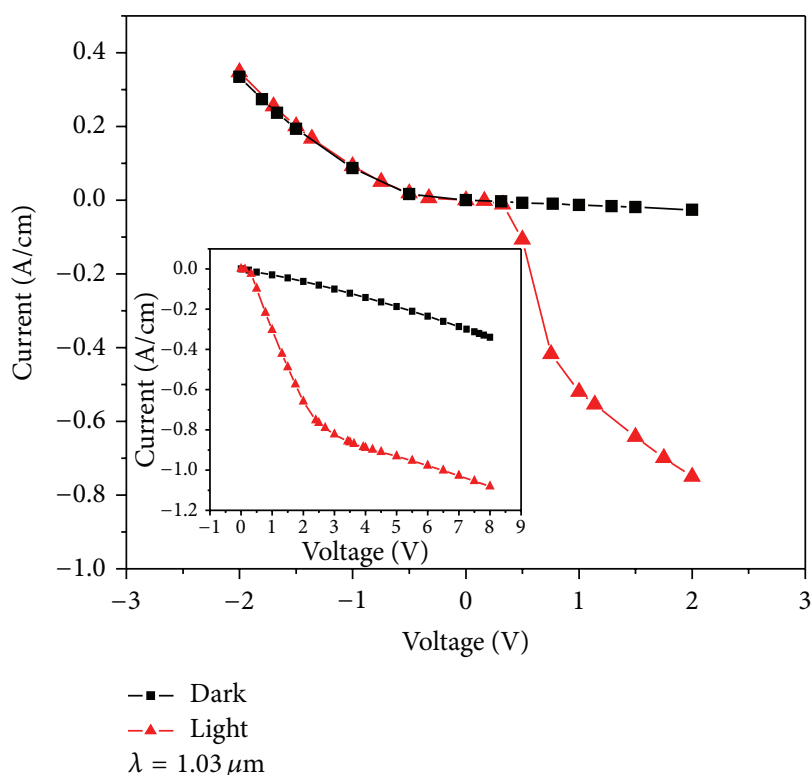

(a)

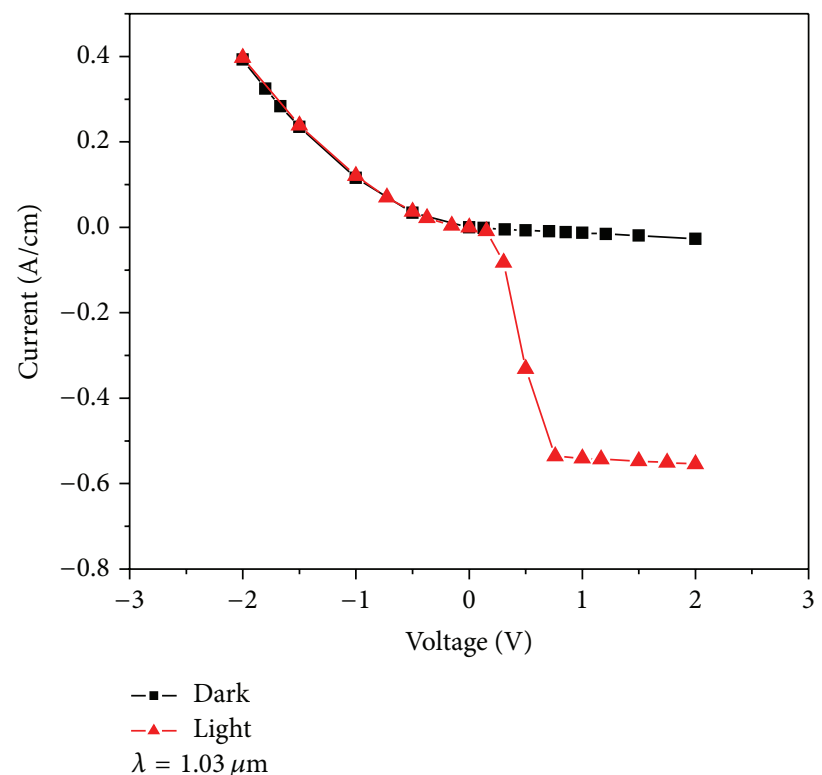

(b)

FIgure 4: (a) Current-voltage characteristics of the InGaAs QD RCE photodetector. The inset shows the $I-V$ characteristics at high bias voltage. (b) Current-voltage characteristics of the InAs QD RCE photodetector.

the main mechanism for photocurrent generation is due to thermal ionization. We also found that as the bias increased from $0.5 \mathrm{~V}$ to $2 \mathrm{~V}$ the photocurrent density increased 1 order of magnitude from $0.02 \mathrm{~A} / \mathrm{cm}$ to $0.34 \mathrm{~A} / \mathrm{cm}$. This rapid increase of photocurrent density is because the interaction of thermal ionization and tunneling ionization caused exciton ionization.

We also simulated $I-V$ characteristic curve at high bias, as shown in Figure 4(a) inset. Photocurrent increases as the bias voltage increases. But when bias voltage exceeds 2 volts, photocurrent stays at about the same value as the bias voltage increases. This is because the majority of excitons are ionized when bias voltage is above 2 volts and thus would not generate photocurrent.

The electric current characteristic $(I-V)$ of InAs QD RCE simulated still to compare with InGaAs QD. But it is seen from Figure 4(b) that the photocurrent reaches saturation under low bias. So, the paper has discussed the effect of the resonant cavity, showing why the InGaAs/GaAs quantum dot is, rather than others quantum $\operatorname{dot}(\mathrm{InAs} / \mathrm{GaAs})$.

The quantum efficiency of a QD-RCE photodetector is determined by the cavity resonant wavelength relative to the exciton absorption peak. When these two wavelengths overlap, the photodetector has the ideal quantum efficiency, 


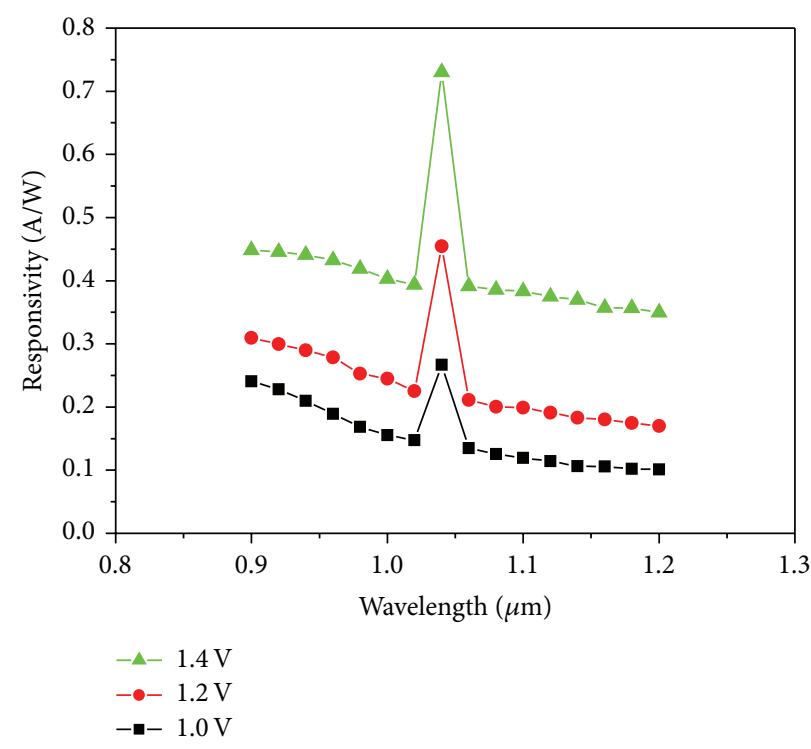

FIGURE 5: Responsivity spectra of the RCE photodetector under different reverse bias voltages.

showing wavelength selectivity. Full width at half maximum (FWHM) can reflect the wavelength selectivity of RCE photodetector. Consider

$$
\mathrm{FWHM}=\frac{\lambda_{0}^{2}\left(1-\sqrt{R_{1} R_{2}} e^{-\xi \alpha d}\right)}{2 \pi \eta_{g} L_{\mathrm{eff}} \sqrt[4]{R_{1} R_{2}} e^{-\xi \alpha d / 2}}
$$

Here, $R_{1}$ and $R_{2}$ are the reflection coefficients of the top DBR and the bottom DBR, respectively. $\alpha$ is the absorption coefficient and $d$ the thickness of the absorption layer. $L_{\text {eff }}$ is the equivalent cavity length, $n_{g}$ is the group refraction rate, $\lambda_{0}$ is the mode wavelength, and $\xi$ is the standing wave enhancement factor.

The device photocurrent spectra at different bias voltages were simulated. Figure 5 shows the spectral responsivity simulation of the RCE photodetector under different applied reverse bias voltages. The resonant peak wavelength was at $1030 \mathrm{~nm}$ with $3 \mathrm{~nm}$ full width at half maximum (FWHM). The responsivity increases with the applied reversed bias between $0 \mathrm{~V}$ and $3 \mathrm{~V}$ and maintains the same shape. The responsivity is approximately $0.25 \mathrm{~A} / \mathrm{W}$ at $1.0 \mathrm{~V}$ bias and increases to $0.73 \mathrm{~W} / \mathrm{A}$ upon $1.4 \mathrm{~V}$ bias and the quantum efficiency is $30.1 \%$ and $87.9 \%$, respectively, which agrees with the reported results in [12].

Figure 6(a) shows the $C-V$ simulation performed at $F=$ $100 \mathrm{KHz}, 1 \mathrm{MHz}$, and $10 \mathrm{MHz}$ in InGaAs QDs. More carriers will extend across the QDs and the excited state and the ground state will be filled with increasing reverse bias. So, the higher the reverse bias, the larger the capacitance value, as a result of the greater portion of the QD assembly being populated [14-17]. We also simulated $C-V$ characteristic of InAs QD RCE to compare with InGaAs QD as shown in Figure 6(b). The photocurrent has reached saturation at 1.0 V. It is similar to Figure 4(b), the $I-V$ characteristic. The theoretical fit of the capacitance is obtained using a simple model based on the definition of the capacitance $(C=$ $d Q / d V)[18]$ where the charge is given by the integral of the density of states and the energy distribution:

$$
C_{\mathrm{dot}}=q A L \sum_{i} \frac{\partial}{\partial V_{\mathrm{dot}}}\left(\int D_{i}\left(E, V_{\mathrm{dot}}\right) \times f\left(E, V_{\mathrm{dot}}\right) d E\right)
$$

The summation is assumed over all $i$ subbands, where $D_{i}\left(E, V_{\text {dot }}\right)$ is the electron density of state for $i$ th subband in QDs, $f\left(E, V_{\text {dot }}\right)$ is the Fermi-Dirac energy distribution, $V_{\text {dot }}$ is the voltage across the quantum dots scaled by level-arm relation when the band bending is neglected, and $L$ is the level arm coefficient.

Comparing with $90.3 \%$, the experimentally measured results for quantum efficiency and the discrepancy in the device responsivity in our computed result can be attributed to the number of quantum dots used in simulation. The device in the experimental result has 22 pairs of QDs, whilst in simulation the lowest quantum dot number allowed was limited by the calculation procedure. And to some extent, this result further demonstrated QD RCE-PD's potential in achieving high quantum efficiency, high response rate, and better wavelength selectivity.

\section{Conclusions}

In summary, we have simulated an n-i-n RCE photodetector grown on a GaAs substrate with operating wavelength at $1.03 \mu \mathrm{m}$. The responsivity is $0.73 \mathrm{~A} / \mathrm{W}$ at $1.4 \mathrm{~V}$ bias with a FWHM of $3 \mathrm{~nm}$. A high quantum efficiency of $87.9 \%$ was observed in the calculation. The device has reduced dark current of $0.0034 \mathrm{~A} / \mathrm{cm}$ at the bias of $0 \mathrm{~V}$ and $0.026 \mathrm{~A} / \mathrm{cm}$ at the reverse bias of $2.0 \mathrm{~V}$. 


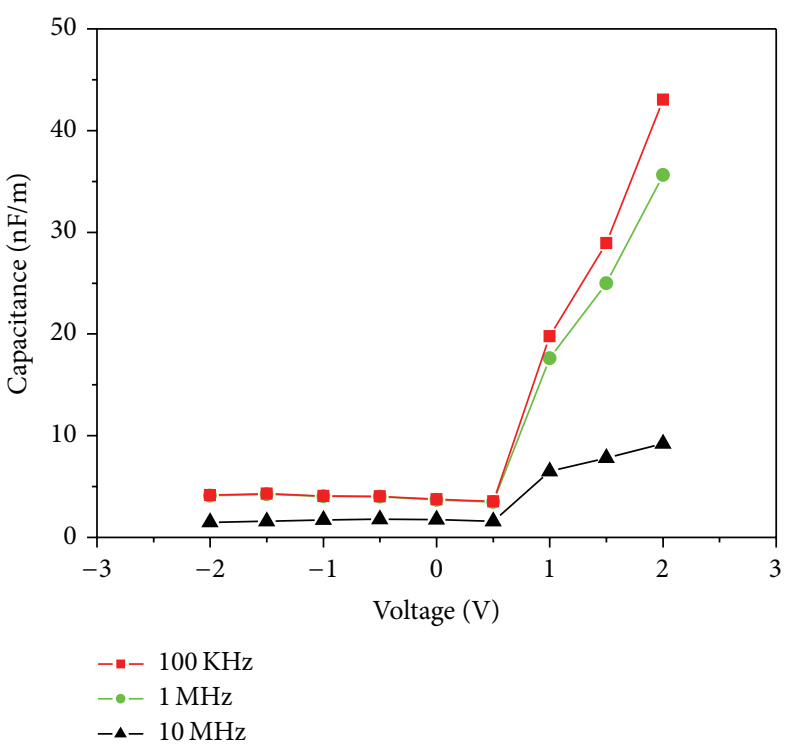

(a)

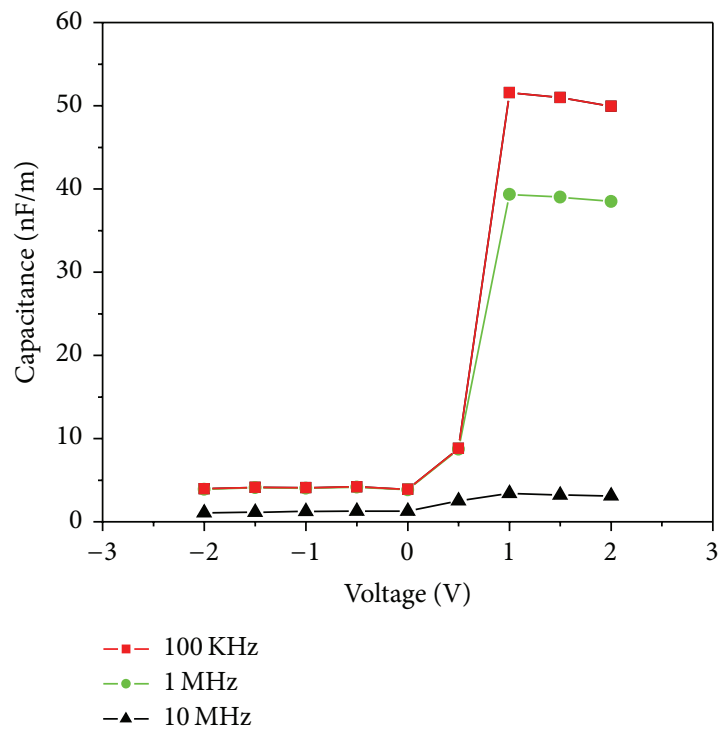

(b)

FIGURE 6: (a) $C-V$ simulation figure of the InGaAs QD RCE photodetector under different frequents. (b) $C$ - $V$ characteristics of the InAs QD RCE photodetector.

\section{Conflict of Interests}

The authors declare that there is no conflict of interests regarding the publication of this paper.

\section{Acknowledgments}

This work was supported by National Scientific Research Plan (2011CB932903) and State Scientific and Technological Commission of Shanghai (no. 118014546) and State Key Laboratory of Functional Materials for Informatics and National Laboratory for Terahertz Solid State Technology, Chinese Academy of Science.

\section{References}

[1] A. Shen, H. C. Liu, M. Gao et al., "Resonant-cavity-enhanced p-type GaAs/AlGaAs quantum-well infrared photodetectors," Applied Physics Letters, vol. 77, no. 15, pp. 2400-2402, 2000.

[2] A. Ramam, G. K. Chowdhury, and S. J. Chua, "An approach to the design of highly selective resonant-cavity-enhanced photodetectors," Applied Physics Letters, vol. 86, Article ID 171104, 3 pages, 2005.

[3] Q. Han, X. H. Yang, Z. C. Niu et al., "1.55 $\mu \mathrm{m}$ GaInNAs resonant-cavity-enhanced photodetector grown on GaAs," Applied Physics Letters, vol. 87, no. 11, Article ID 111105, 3 pages, 2005.

[4] S. Q. Liu, Q. Han, B. Zhu et al., "High-performance metamorphic InGaAs resonant cavity enhanced photodetector grown on GaAs substrate," Applied Physics Letters, vol. 98, no. 20, Article ID 201104, 3 pages, 2011.

[5] Q. Han, Z. C. Niu, L. H. Peng et al., "High-performance $1.55 \mu \mathrm{m}$ low-temperature-grown GaAs resonantcavity-enhanced photodetector," Appliec Physics Letters, vol. 89, Article ID 131104, 3 pages, 2006.
[6] N. Guo, W. Hu, X. Chen, L. Wang, and W. Lu, "Enhanced plasmonic resonant excitation in a grating gated field-effect transistor with supplemental gates," Optics Express, vol. 21, no. 2, pp. 1606-1614, 2013.

[7] M. Casalino, G. Coppola, M. Gioffr et al., "Microcavity silicon photodetectors at $1.55 \mu \mathrm{m}$," Advances in OptoElectronics, vol. 2011, Article ID 965967, 10 pages, 2011.

[8] O. Baklenov, H. Nie, K. A. Anselm, J. C. Campbell, and B. G. Streetman, "Multi-stacked quantum dot resonant-cavity photodetector operating at $1.06 \mu \mathrm{m}$," Electronics Letters, vol. 34, no. 7, pp. 694-695, 1998.

[9] H. Nie, O. Baklenov, P. Yuan, C. Lenox, B. G. Streetman, and J. C. Campbell, "Quantum-dot resonant-cavity separate absorption, charge, and multiplication avalanche photodiode operating at $1.06 \mu \mathrm{m}$," IEEE Photonics Technology Letters, vol. 10, no. 7, pp. 1009-1011, 1998.

[10] H. Zhang, H. Z. Zheng, P. Xu et al., "Tuning of detection wavelength in a resonant-cavity-enhanced quantum-dotembedded photodiode by changing detection angle," Chinese Physics Letters, vol. 22, no. 6, pp. 1405-1408, 2005.

[11] A. J. Bennett, D. C. Unitt, P. See et al., "Microcavity singlephoton-emitting diode," Applied Physics Letters, vol. 86, no. 18, Article ID 181102, 3 pages, 2005.

[12] X. M. Sun, H. Zhang, H. Zhu et al., "High responsivity resonantcavity-enhanced InGaAs/GaAs quantum-dot photodetector for wavelength of $\sim 1 \mu \mathrm{m}$ at room temperature," Electronics Letters, vol. 45, no. 6, pp. 329-331, 2009.

[13] J. C. Campbell, D. L. Huffaker, H. Deng, and D. G. Deppe, "Quantum dot resonant cavity photodiode with operation near $1.3 \mu \mathrm{m}$ wavelength," Electronics Letters, vol. 33, no. 15, pp. 13371339, 1997.

[14] H. Zhang, H.-Z. Zheng, P. Xu et al., "Tuning of detection wavelength in a resonant-cavity-enhanced quantum-dot-embedded photodiode by changing detection angle," Chinese Physics Letters, vol. 22, no. 6, pp. 1405-1408, 2005. 
[15] O. Saad, M. Baira, R. Ajjel et al., "Capacitance-voltage analysis of InAs quantum dots grown on InAlAs/InP(001)," Microelectronics Journal, vol. 39, no. 1, pp. 7-11, 2008.

[16] G. R. Li, X. Zhou, F. H. Yang, P. H. Tan, H. Z. Zheng, and Y. P. Zeng, "Photo-capacitance response of internal tunnelling coupling in quantum-dot-imbedded heterostructures under selective photo-excitation," Journal of Physics Condensed Matter, vol. 16, no. 36, pp. 6519-6525, 2004.

[17] W. Lu, Y. M. Mu, X. Q. Liu et al., "Direct observation of abovequantum-step quasibound states in GaAs/AlxGal-xAs/vacuum heterostructures," Physical Review B-Condensed Matter and Materials Physics, vol. 57, no. 16, pp. 9787-9791, 1998.

[18] G. Medeiros-Ribeiro, F. G. Pikus, P. M. Petroff, and A. L. Efros, "Single-electron charging and Coulomb interaction in InAs self-assembled quantum dot arrays," Physical Review BCondensed Matter and Materials Physics, vol. 55, no. 3, pp. 15681573, 1997. 

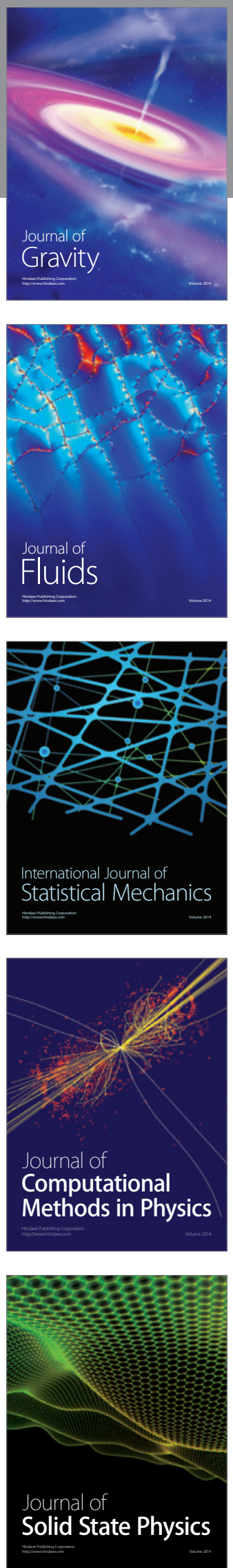

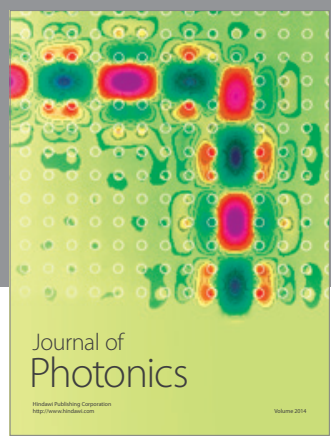

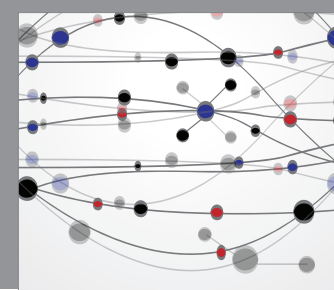

The Scientific World Journal

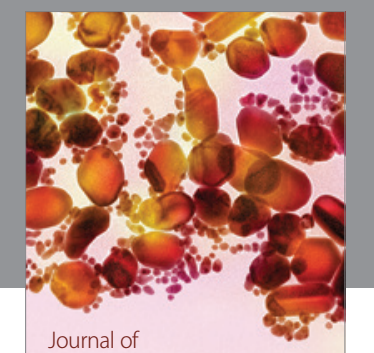

Soft Matter
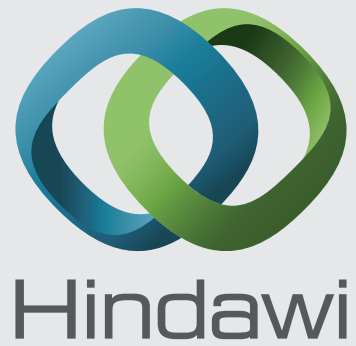

Submit your manuscripts at

http://www.hindawi.com
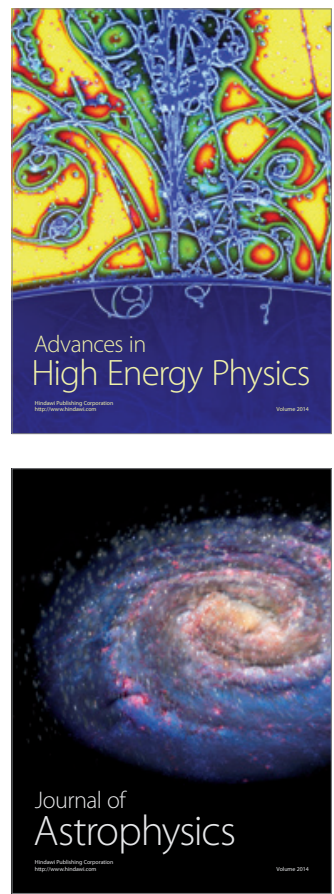
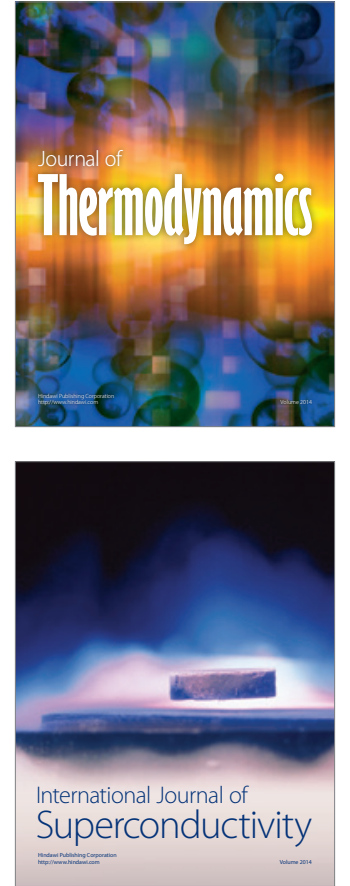
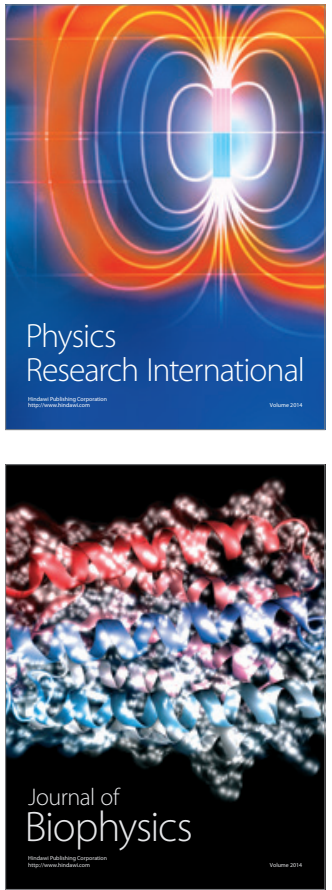
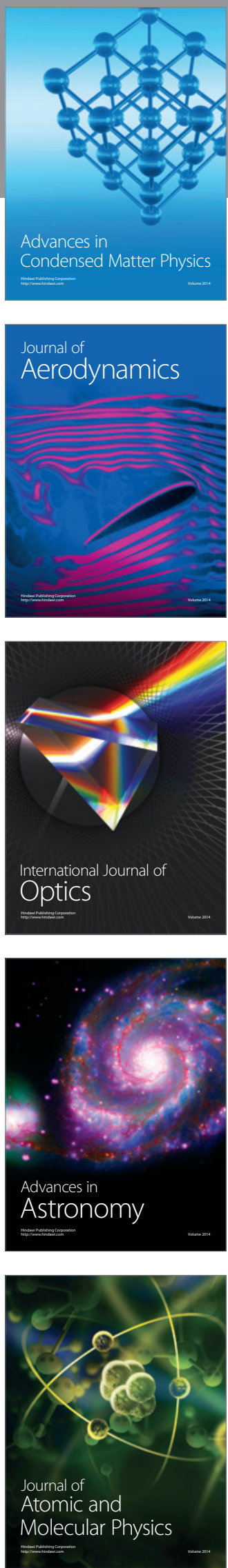